\title{
Significant differences in terms of codon usage bias between bacteriophage early and late genes: a comparative genomics analysis
}

\author{
Oriah Mioduser $^{1 \dagger}$, Eli Goz ${ }^{1,2+}$ and Tamir Tuller ${ }^{1,2,3^{*}}$
}

\begin{abstract}
Background: Viruses undergo extensive evolutionary selection for efficient replication which effects, among others, their codon distribution. In the current study, we aimed at understanding the way evolution shapes the codon distribution in early vs. late viral genes in terms of their expression during different stages in the viral replication cycle. To this end we analyzed 14 bacteriophages and 11 human viruses with available information about the expression phases of their genes.

Results: We demonstrated evidence of selection for distinct composition of synonymous codons in early and late viral genes in 50\% of the analyzed bacteriophages. Among others, this phenomenon may be related to the time specific adaptation of the viral genes to the translation efficiency factors involved at different bacteriophage developmental stages. Specifically, we showed that the differences in codon composition in different temporal gene groups cannot be explained only by phylogenetic proximities between the analyzed bacteriophages, and can be partially explained by differences in the adaptation to the host tRNA pool, nucleotide bias, GC content and more. In contrast, no difference in temporal regulation of synonymous codon usage was observed in human viruses, possibly because of a stronger selection pressure due to a larger effective population size in bacteriophages and their bacterial hosts.

Conclusions: The codon distribution in large fractions of bacteriophage genomes tend to be different in early and late genes. This phenomenon seems to be related to various aspects of the viral life cycle, and to various intracellular processes. We believe that the reported results should contribute towards better understanding of viral evolution and may promote the development of relevant procedures in synthetic virology.
\end{abstract}

Keywords: Viral evolution, Codon usage bias (CUB), Bacteriophage genome evolution, Viral life cycle, Coding regions, Synthetic virology

\section{Background}

Deciphering the regulatory information encoded in the genomes of phages and other viruses, and the relation between the nucleotide composition of the coding regions and the viral fitness is of great interest in recent years.

* Correspondence: tamirtul@post.tau.ac.il

${ }^{\dagger}$ Equal contributors

${ }^{1}$ Department of Biomedical Engineering, Tel-Aviv University, Ramat Aviv, Israel

${ }^{2}$ SynVaccineLtd. Ramat Hachayal, Tel Aviv, Israel

Full list of author information is available at the end of the article
Gene expression within different Deoxy ribonucleic Acid (DNA) viruses or viruses with DNA intermediate, such as herpeses, lenti-retro, polyoma, papilloma, adeno, parvo and various families of bacteriophages is regulated in a temporal fashion and can be divided into early and late stages with respect to the viral replication cycle [1-8].

The early genes are expressed following the entry into the host cell and code typically for non-structural proteins that are responsible for different regulatory functions in processes such as: viral DNA replication, activation of late genes expression, trans-nuclear 
transport, interaction with the host cell, induction of the cell's DNA replication machinery necessary for viral replication, etc. $[9,10]$. Late genes largely code for structural proteins required for virion assembly; they are generally highly expressed and their expression is usually induced or regulated by the early genes $[9,10]$.

Several studies have shown that viral codon frequencies tend to undergo evolutionary pressure for specific CUB; among others, it was suggested that viral CUB is under selection for improving the viral fitness, and in specifically the viral gene expression [11-33].

In particular, in [17] different trends of translation efficiency adaptation of the coding regions of the bacteriophage Lambda early and late genes were demonstrated. Specifically, it was shown that the preferences of codons in early genes, but not in the late genes, were similar to those of the bacterial host [17]. The analysis of ribosome profiling data revealed that the codon decoding rates of viral genes tend to correlate with their expression levels [17]. Interestingly, during the initial stages of phage development the decoding rates in early genes were found to be higher than the decoding rates in late genes; in more progressive viral cycles an opposite trend was demonstrated [17].

In this study we go further, and perform a comparative genomics analysis of the temporal differences in CUB in almost all known viruses with existing in the literature classification of their genes into early and late groups. Specifically basing on analysis of 14 bacteriophages and 11 human viruses we suggest that $50 \%$ of the analyzed bacteriophages tend to undergo an extensive evolutionary selection for distinct compositions of synonymous codons in early and late viral genes. We analyze the features of the genomes that undergo this type of selection and argue that the differential CUB can be related to various intracellular phenomena and processes, such as: translational selection and regulation $[11,12,17,21,22,28,31]$, mutational bias and pressure [16, 20, 21, 23, 26, 27, 30, 32, 33], amino acids (AA) compositions [12, 16], and other genomic characteristics, some of which are still not fully understood [13, 14, 29, 34].

Finally, we discuss a possible application of our findings to synthetic virology. Specifically, we suggest using the temporally regulated CUB for controlling the viral gene expression at different time points during the life cycle for designing of optimized and/or deoptimized synthetic viruses which can be used in exploring novel strategies in vaccination (e.g. life attenuated vaccines) and cancer therapy (oncolytic viruses).

\section{Results}

The research outline of the study is described in Fig. 1. More details can be found in the following sections.
Bacteriophage early and late genes tend to have different compositions of synonymous codons

Genome level information about the different viruses analyzed in this study, like their hosts, number of genes, gene lengths and ENC, is displayed in Additional file 1: Table S1 and Figure S1.

In order to compare the synonymous codons usage in early and late genes, each coding sequence was represented by its relative synonymous codons frequencies (RSCF) - a 61 dimensional vector expressing each sense codon by its frequency in that sequence normalized relative to the frequencies of other synonymous codons coding for the same AA. We then performed a clustering analysis, assuming that RSCF vectors that are closer with respect to Euclidian metric correspond to genes with a more similar content of synonymous codons (see Materials and Methods).

Our results suggest that early and late genes in $50 \%$ of the analyzed bacteriophages tend to exploit different synonymous codons. Specifically, in 7 of the 14 analyzed bacteriophages, early and late genes were found to be significantly ( $p$-value $\leq 0.05$ ) separated according to the frequencies of their synonymous codons (Figs. 2, 3a, b, Additional file 1: Figure S2 and Figure S3 in Section 1.2). Our analysis provide evidence that different sets of synonymous codons in early vs. late genes are selected for in the course of viral evolution; these differences may be related to the optimization of bacteriophage fitness in different phases of the viral lifecycles.

In addition, 6 out of 14 bacteriophages were also found to be significantly ( $p$-value $<0.05)$ separated according to the AA composition of their early and late genes (Fig. 3b, Additional file 1: Figure S4 and Figure S5 in Section 1.2). 4 viruses were characterized both by a differential synonymous codon usage and by a differential AA usage in their early and late genes. These findings suggest that among others, the different codon distribution in early and late genes may be partially related to the functionality of the encoded proteins via their AA content and possibly protein folding [35].

To check if bacteriophages with significant differences in synonymous codons usage in temporal genes tend to have more similar genomic sequences (usually related to smaller evolutionary distances), we reconstructed a phylogenetic tree of the bacteriophage proteomes based on Average Repetitive Subsequences (ARS) distance matrix and neighbor joining method as described in Materials and Methods section and in references therein (Fig. 3a). We then performed a statistical analysis in order to investigate the relation between the differences in temporal regulation of synonymous codons in different viruses and their evolutionary distances. We did not find such a relation 


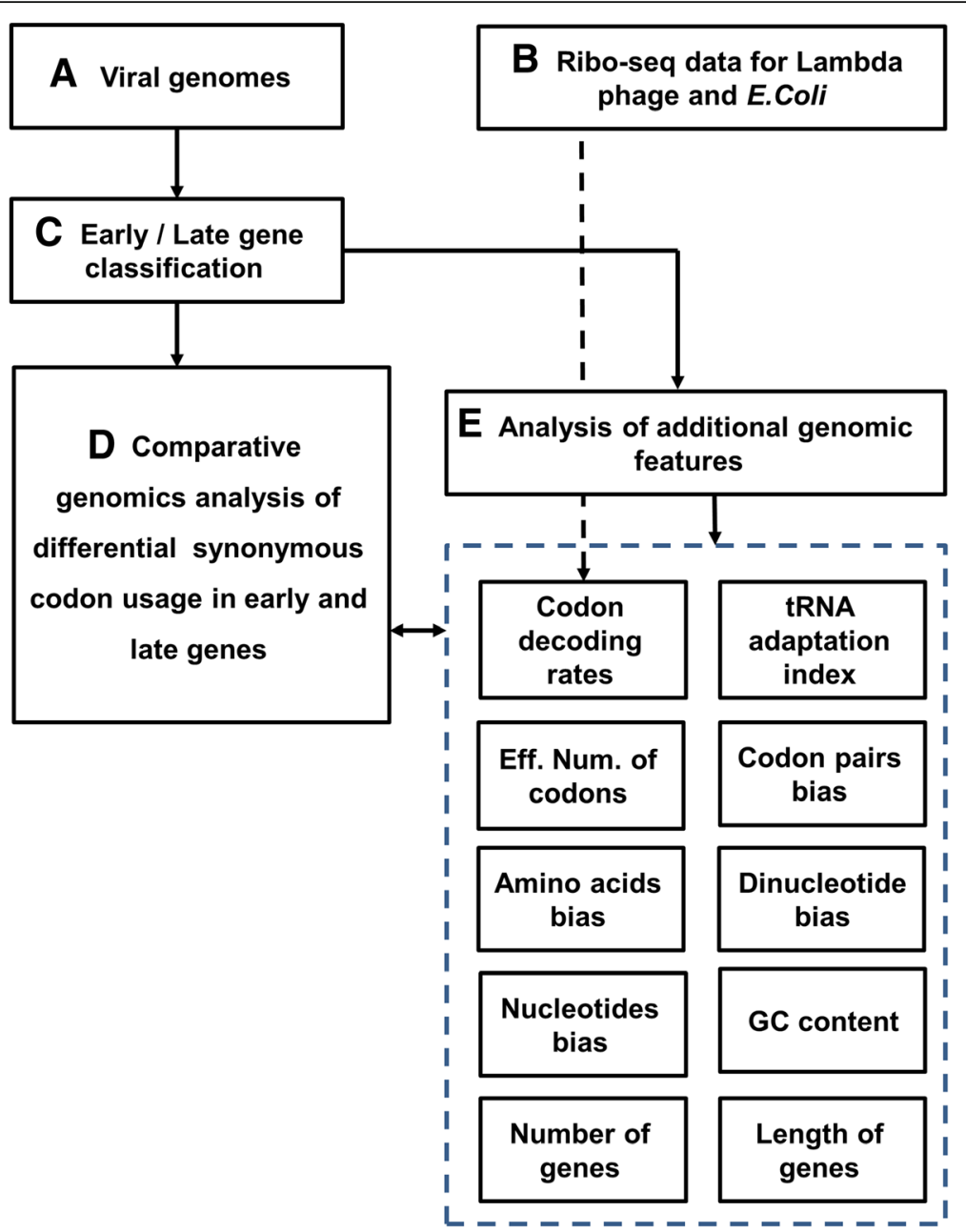

Fig. 1 The research outline of the study. The details can be found in the main text: Our analysis was based on coding sequences of 14 bacteriophages and 11 human viruses (A.), and on the ribo-seq measurements of bacteriophage Lambda and its E.coli host (B.). Basing on the existing literature, classification of the viral genes to early and late (with respect to the beginning of the lytic phase) was derived (C.). A., B., and C, were used to perform a comprehensive comparative genomics analysis of differential synonymous codon usage in early and late genes (D.), as well as of additional genomic features possibly related to codon bias (E.), such as: ribo-seq based codon typical decoding rates (TDR), Transfer Ribonucleic Acid (tRNA) adaptation indexes (tAl), effective number of codons (ENC), codon pairs bias (CPB), amino acids bias (AAB), dinucleotide bias (DNTB), nucleotide bias (NTB), GC content, number of genes in each temporary group and their length

(see details in Additional file 1: Section 1.3 and Figure S6), suggesting that the differential codon usage in early and late genes is a complex trait related to alternative determinants such as the bacterial niche, the specific phage proteins and their function/ structure, etc.

Viruses undergo an extensive evolutionary selection for adaptation to their host's cell environment, and thus it can be assumed that their codon composition reflects an efficient adaptation of the viral genes to specific intracellular conditions (e.g. in terms of gene expression factors such as tRNA molecules, AA concentration, etc) that are prevalent in different gene expression stages, in accordance with the reported results.

\section{Weaker separation between synonymous codon usage in} early and late genes in human viruses

The results in the previous section suggest that bacteriophages undergo an extensive evolutionary selection on a synonymous level for temporal regulation of gene expression. Whether this also occurs in viruses of humans and other eukaryotic hosts is harder to ascertain. Human Immunodeficiency Virus 1 (HIV-1) was found to have a significant separation ( $p$-value $\leq 0.05)$ of codon composition between early and late genes, while such separation was not statistically significant in the rest of the analyzed viruses (see Additional file 1: Table S2 in Section 1.4).

As evidenced in Additional file 1: Table S1 and Figure S1, human viruses tend to have fewer genes than 
T4

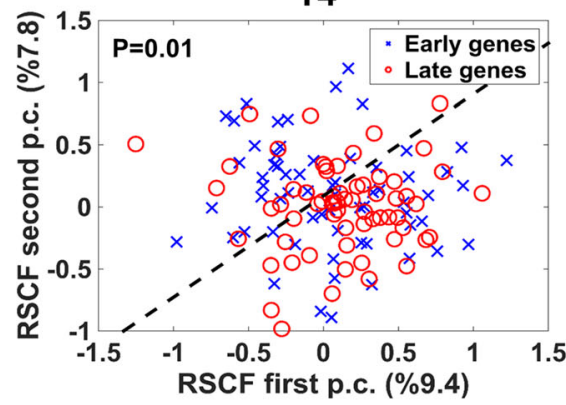

T7

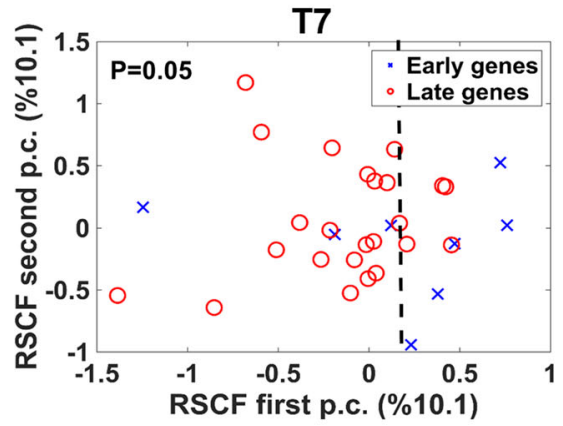

$\mathrm{Mu}$

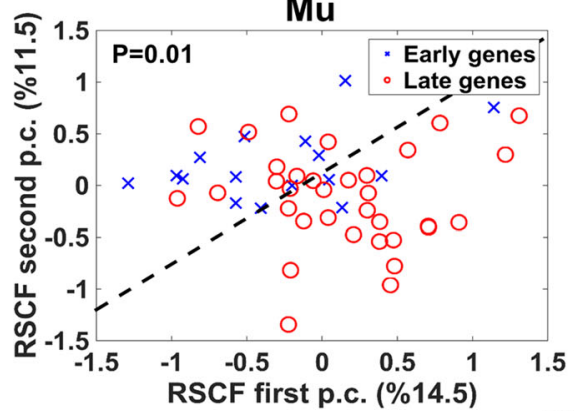

Pak-P3

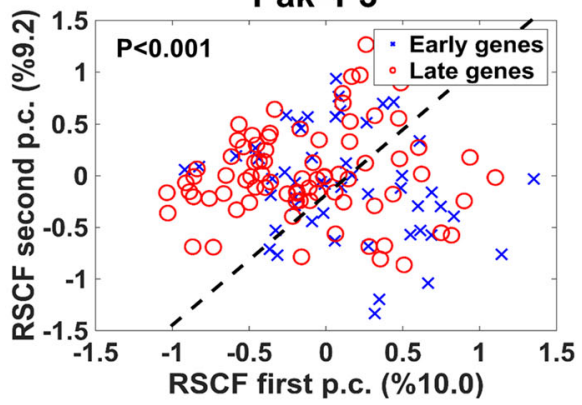

PhiYs40

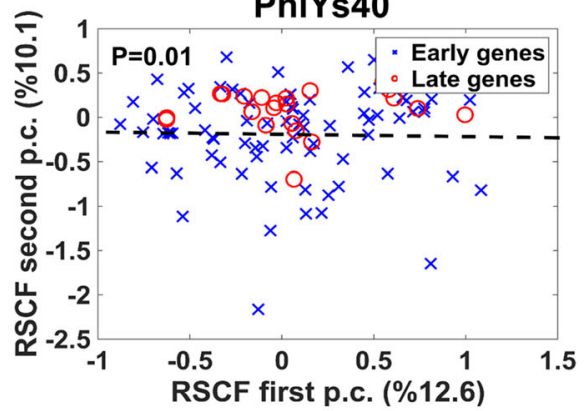

P2345

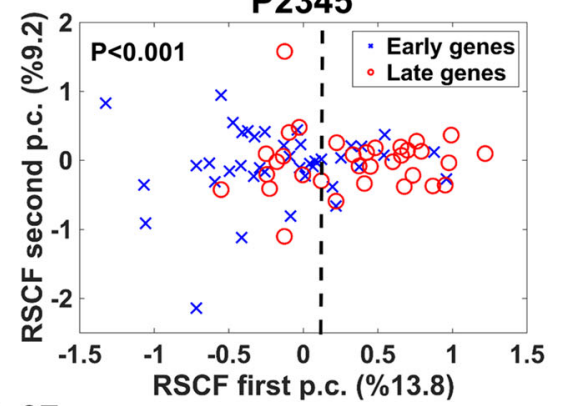

PhiR1-37

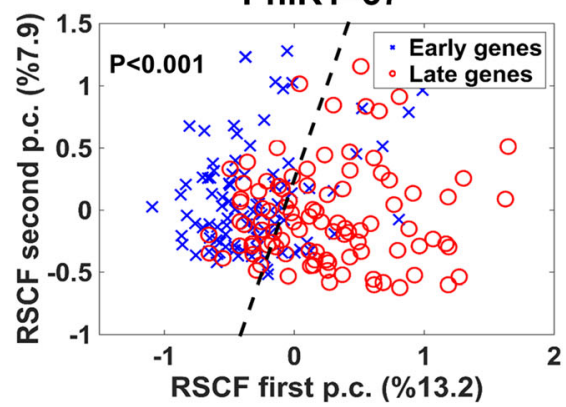

Fig. 2 Principal component analysis (PCA) of RSCF vectors for bacteriophages with significant separation in codon usage between early (blue circles) and late (red circles) genes. In order to visualize the clustering, PCA was applied to project the RSCF vectors to a plane spanned by their first two principal components. In order to visualize the separation between clusters a maximum margin separation line, a line for which the Euclidian distance between it and the nearest point from either of the groups is maximized, was calculated and plotted. The significance of cluster separation was assessed by comparing the Davies-Bouldin cluster score to the randomized scores obtained from 100 permutations of gene temporary (early or late) labels. The variances \% of the first two principal components are mentioned in the figures axis

bacteriophages. Therefore, we were interested in checking whether this fact can explain the weaker signal for temporal separation in CUB, and if, in practice, human viruses may also behave as bacteriophages with respect to the differential usage of synonymous codons in their early and late genes. To this end we analyzed the 7 bacteriophages with temporary differential codon usage by sampling in each one of them a number of early and late genes that is typical to human viruses (average of 8 early genes and 14 late genes). We found that the 


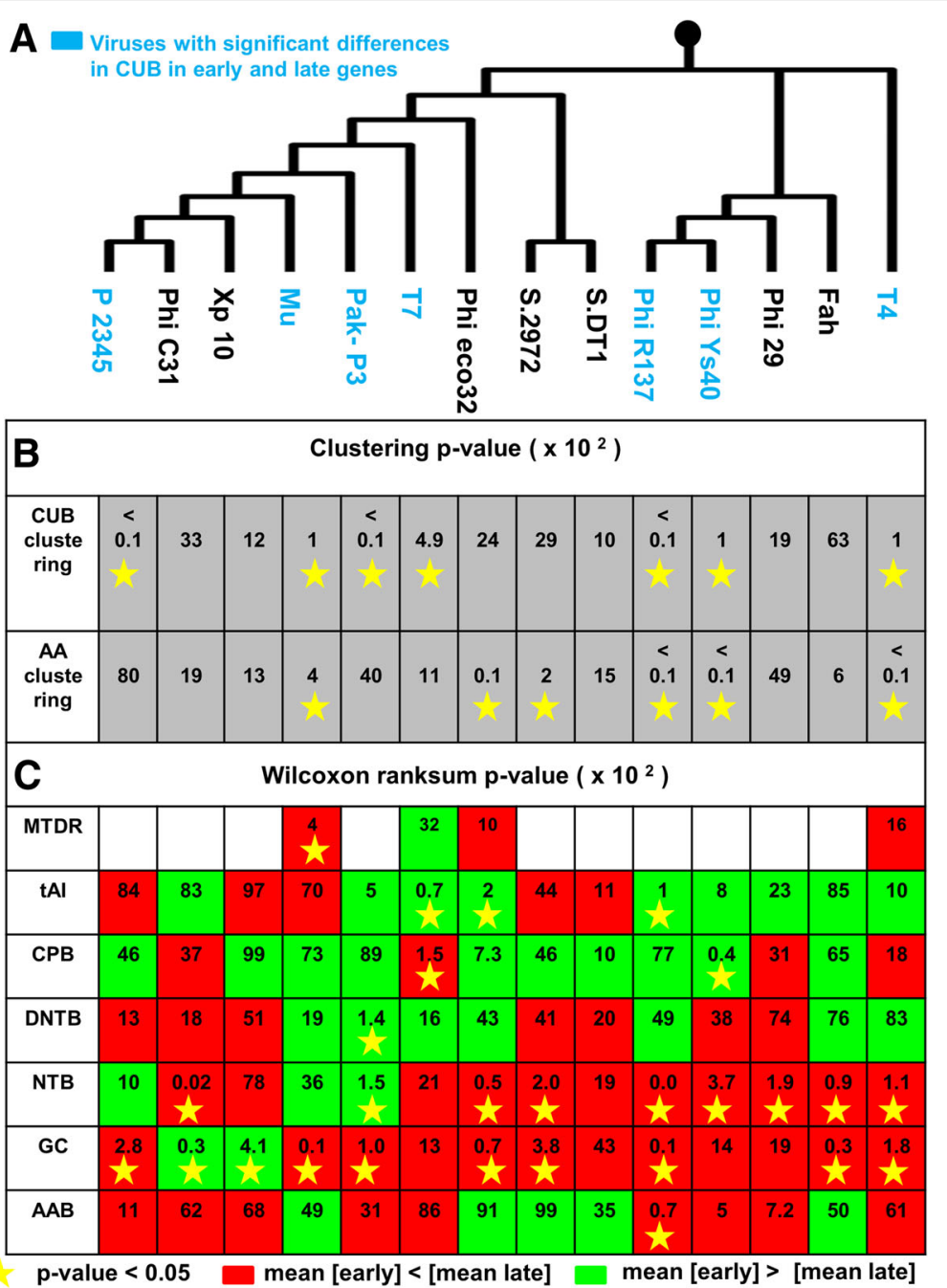

Fig. 3 Comparative analysis of early and late genes in 14 different bacteriophages. Details can be found in the main text. a A phylogenetic tree built from complete phage proteomes using ARS distance (see Materials and Methods). Phages with significant differences in temporary codon usage are marked by blue. $\mathbf{b}$ Viruses with significant $(p$-value $<0.05)$ separation between early and late genes w.r.t synonymous codons or AA are marked by yellow stars. c Significance of separation between early and late genes w.r.t additional genomic features estimated by Wilcoxon ranksum $p$-value. Features/viruses with significant ( $p$-value $<0.05$ ) separation between the two temporal groups are marked by yellow stars; green is related to higher mean in the case of the early genes and red is related to higher mean in the case of the late genes

temporal differences in codon usage remained significant even after randomly reducing the number of genes, indicating, among others, that these differences cannot be directly explained only by the genome size.

\section{Comparison of early and late genes with respect to additional features of their coding regions}

The signal of selection for temporarily regulated composition of synonymous codons in bacteriophages demonstrated in the previous subsection led us to analyze additional genomic features, such as: codon mean typical decoding rate (MTDR), tRNA adaptation index (tAI), codon pairs bias (CPB), dinucleotide bias (DNTB), nucleotide bias (NTB), GC content and amino acids bias (AAB).

Various studies related these features to different genomic mechanisms and biological processes involved in viral replication cycles and are related to the viral fitness.

For example, it was suggested that gene translation efficiency can be affected not only by single codons, but also by distribution of codon pairs [36]. In [37-39] it was argued that pairs of adjacent nucleotides may be an important genomic characteristic being under a significant evolutionary pressure in viruses and their hosts; specifically, it was suggested that CpG pairs are underrepresented in many Ribonucleic Acid (RNA) and in 
most small human DNA viruses, in correspondence to dinucleotide frequencies of their hosts. This phenomenon can be related, for example, to the contribution of the CpG stacking basepairs to RNA folding [40] and/or to the enhanced innate immune responses to viruses with elevated CpG [41]. The stability of the RNA secondary structures can be also affected by the genomic composition of nucleotides and in particular by GC content [42]. In addition, nucleotide compositions and AA usage bias may affect, among others, the synthesis of viral molecules, and the function and structure of the encoded proteins.

Consequently, we estimated the listed above features for all genes in all viruses, and evaluated the separation between early and late genes with respect to each one of them (see Materials and Methods). The results shown in Fig. 3c suggest that the differential usage of synonymous codons in early and late genes can be partially related to temporal differences in various characteristics of genomic sequences. Specifically, the features with the strongest temporal differences are the NTB and GC content which are significant $(p$-value $<0.05)$ in most of the phages.

In addition, we wanted to check if the bacteriophages with a significant temporal separation with respect to synonymous codons tend also to be enriched with specific genomic features in comparison to the group of bacteriophages with non-significant temporal differences in synonymous codons. To this end, we compared the distribution of various genomic features in the two groups. Based on Wilcoxon ranksum test we found no significant differences between the two groups of bacteriophages in terms of: genome length $(p$-value $=0.53), \mathrm{ENC}(\mathrm{p}$-value $=0.4), \mathrm{CPB}(\mathrm{p}$-value $=0.99)$, DNTB $(\mathrm{p}$-value $=0.21), \mathrm{NTB}(\mathrm{p}$-value $=0.9), \mathrm{GC}$ content $(\mathrm{p}$-value $=0.8)$ and $\mathrm{AAB}(\mathrm{p}$-value $=0.99)$. See also Additional file 1: Figure S7 in Section 1.5.

\section{Discussion}

In this study, we performed a comparative genomics analysis of viruses with annotations in literature regarding their genes division according to temporal expression. We examined 14 bacteriophages with different bacterial hosts and 11 human viruses in order to understand if there is a universal difference in synonymous codons usage as well as in additional genomic features (such as codon decoding rates, nucleotide/dinucleotide/ AA biases, GC content and others) with respect to different temporal stages of viral life cycle.

Our results suggest that $50 \%$ of bacteriophages undergo an extensive evolutionary selection for distinct compositions of synonymous codons in early and late viral genes. This phenomenon was found to be weaker/ less significant in human viruses, possibly because of the stronger selection pressure in bacteriophages / bacteria due to the larger size of their populations, and because of the fact that regulation processes in human gene expression are more 'complex' and thus may be mediated by additional aspects not necessary related to codons.

The differences between early and late genes, both with respect to the composition of synonymous codons and with respect to additional genomic features described in the previous sections, can be possibly influenced by various intracellular phenomena and processes related to the optimization of gene expression and to the overall fitness of the phage. To mention a few, these phenomena/processes include: adaptation of translation elongation efficiency in different phases of the viral lifecycle [17], Messenger Ribonucleic Acid (mRNA) folding $[43,44]$, adaptation of the viral genes to the (possibly altering) tRNA pool of their hosts $[11,12,17,31]$, mutation levels and biases $[16,20,21,23,26,27,30,32,33]$, transcription regulation $[45,46]$, protein function and structure [47], cell metabolism [48], etc.

There can be various explanations to the fact that it seems that only $50 \%$ of the bacteriophages there is a significant difference in the codon usage in early vs. late genes:

First, it is possible that the effective population size (which is not easy to estimate) varies among the analyzed bacteriophages. The selection pressure is weaker in bacteriophages with smaller population size.

Second, this observation may be also related to the intracellular regimes during the development of the different bacteriophages. For example, it is possible that during the development of some bacteriophages the tRNA levels are modulated/changed, while in other cases the changes are minor. The changes in the tRNA levels may trigger evolution of different CUB in early/late genes in the bacteriophages that experience them.

Third, this result may be related to the nature of the protein encoded in the bacteriophages genome. The specific function and properties of the proteins in different bacteriophages may affect the observed levels of selection. For example, it is possible that only in some bacteriophages the early vs. late genes tend to have different structure with different co-translational folding constraints that eventually affect the codon bias. It is also possible that only in some bacteriophages the early vs. late genes tend to have different expression levels/patterns that eventually affect their codon bias.

It is possible that the results reported here have relevant practical applications. For example, vaccines, and their discovery, are topics of singular importance in present-day biomedical science; however, the discovery of vaccines has hitherto been primarily empirical in nature requiring considerable investments of time, efforts and resourced. To overcome the numerous pitfalls attributed to the classical vaccine design strategies, more efficient and robust rational approaches based on 
computer-based methods are highly desirable. One direction in designing in-silico vaccine candidates may be based on exploiting the temporally regulated synonymous information encoded in the genomes and investigated in this study for attenuating the viral replication cycle while retaining the wild type proteins. In particular, the result reported here suggest that viral genes can be designed with respect to phase specific temporary regulated gene expression constraints, and this design would result in controllable yields of the corresponding genetic products during a defined time period. To achieve this, codons would be selected with frequencies maximally dissimilar / similar to the set of early or late genes than a random set of genes. See Additional file 1: Section 2 and Figures S8, S9 for more details and examples.

\section{Conclusions}

The codon distribution in large fractions of bacteriophage genomes tend to be different in early and late genes. It seems that various additional genomic features (e.g. NTB and GC content) tend to be associated with this signal. This phenomenon seems to be related to various aspects of the viral life cycle, and to various intracellular processes. A similar signal may be observed in human viruses but it seems significantly less frequent. We believe that the reported results should contribute towards better understanding of viral evolution and may promote the development of relevant procedures in synthetic virology.

\section{Material and methods}

The research outline of the study is described in Fig. 1.

\section{Viruses}

Human Viruses analyzed in this study include Herpes viruses, papilloma viruses, Polyomavirus and HIV.

The analyzed bacteriophages include: bacteriophage Lambda, bacteriophage T4, bacteriophage Pak P3, bacteriophage phi29, bacteriophage T7, bacteriophage phiYs40, bacteriophage Fah, bacteriophage xp10, bacteriophage Streptococcus DT1, bacteriophage Streptococcus 2972, bacteriophage $\mathrm{Mu}$, bacteriophage phiC31, bacteriophage phiEco32, bacteriophage p23-45 and bacteriophage phiR1-37.

These viruses were chosen since they have a known division to early and late genes annotated in the literature, as described in Additional file 1: Table S3.

\section{Synonymous codon usage analysis}

Codon composition of a coding sequence was represented by a 61-dimensional vector of RSCF of each one of 61 coding codons (stop codons are excluded).

Clustering analysis was performed on RSCF vectors of each viral coding sequence. Each viral sequence was assigned a group label corresponding to its temporal expression stage (early/late) (according to the classification known in the literature). The tendency of sequences to cluster according to the codons usage in two different clusters corresponding to their temporal expression stages (early/late) was measured using the DaviesBouldin score (DBS) [49]. This score is based on a ratio of within-cluster and between-cluster distances. The optimal clustering solution has the smallest DBS value.

The significance of cluster separation was assessed by comparing the DBS of the wildtype sequences to the randomized scores obtained from 1000 permutations of gene group labels (early or late).

In addition, a similar analysis was performed on AA frequencies as well.

More details can be found in Additional file 1: Section 3.3.

We decided to use the RSCF, since in this study we are interested in comparing the frequencies of the codons without an a-priory assumption/focus on relative bias of codons; to this aim it is more natural to use the RSCF rather the widely used Relative Synonymous Codons Usage (RSCU) measure [50]. However, these measures are similar, and the same analysis performed with RSCU does not change the final conclusions.

\section{Additional genomic features analyzed in this study} The tRNA adaptation index (tAI) quantifies the adaptation of a coding region to the tRNA pool with parameters describing the different tRNAs copy numbers and the selective constraints on the codon-anti-codon coupling efficiency. Since, currently, these parameters are based on gene expression measurements in a very limited number of organisms, and since the efficiencies of the different codon-tRNA interactions are expected to vary among different species, we used a novel approach proposed in [51] for adjusting the tAI weights to any target organism, without the need for gene expression measurements, basing on an optimization of the correlation between the tAI and a measure of codon usage bias. It is the first time, to our knowledge, that this approach is applied to study tAI in viruses with respect to their hosts. The resulting tAI values were computed by a standalone application [52]. See more details in Additional file 1: Section 3.4.

Effective number of codons (ENC) is a measure that quantifies how far the synonymous codon usage of a gene departs from what is expected under the assumption of uniformity [53]. See more details in Additional file 1: Section 3.5.

GC-content is the percentage of nitrogenous bases on a DNA or RNA molecule that are either guanine or cytosine. See more details in Additional file 1: Section 3.6. 
Codon pair bias (CPB). To quantify the $\mathrm{CPB}$, we follow [54] and define a codon pair score (CPS) as the log ratio of the observed over the expected number of occurrences of this codon pair in the coding sequence. The $\mathrm{CPB}$ of a virus is then defined as an average CPSs over all codon pairs comprising all viral coding sequences. See more details in Additional file 1: Section 3.7.

Dinucleotide bias (DNTB). We define a dinucleotide score (DNTS) for a pair of nucleotides as an observed over expected ratio of its occurrences in a sequence. The DNTB of a virus is defined as an average of DNTSs over all dinucleotides comprising all viral coding sequences. See more details in Additional file 1: Section 3.8.

Nucleotide (NTB) and amino acid (AAB) biases are defined as a normalized Shannon entropy over the frequencies of the nucleotides / AA in a genomic sequence. See more details in Additional file 1: Section 3.9.

\section{Ribosome profiling analysis}

Ribosome profiling (ribo-seq) data was taken from [55]. Ribosome profiles for bacteriophage Lambda and Escherichia coli (E.Coli) were reconstructed and normalized as in [17]. The normalization enables measuring the relative time a ribosome spends translating each codon in a specific gene relative to other codons, while considering the total number of codons in this gene, and results in codons normalized footprint count (NFC).

Codon typical decoding rate (TDR). Following [17], in order to estimate the typical decoding time of each codon based on the corresponding ribo-seq data, we used a novel statistical model [56] which takes into consideration the skewed nature of the NFC distribution and describes the NFC histogram of each codon as an output of a random variable which is a sum of a normally distributed and an exponentially distributed random variables called Exponentially Modified Gaussian (EMG). Maximum likelihood criterion was used to estimate the parameters of these distributions for each codon according to the ribo-seq data by fitting the suggested model to the NFC distribution. The mean of the normal distribution component of EMG was called $\mu$, and $\frac{1}{\mu}$ was defined to be the TDR of a codon [17]. See more details in Additional file 1: Section 3.10.

Mean typical decoding rate (MTDR) is a measure which estimates the global translation elongation efficiency of the entire gene as a geometric average of TDRs of its codons. See more details in Additional file 1: Section 3.11.

Since bacteriophage Lambda is the only phage with publicly available ribo-seq data, a direct analysis of TDRs of other phages is currently impossible. Nevertheless, due to the adaptation of the viruses to the translation machinery of their hosts, a rough estimation of MTDR values for other E.Coli phages rather than Lambda may be obtained from the available ribose-seq of the host genes.

\section{Phylogenetic reconstruction}

Following [57], a phylogenetic reconstruction of bacteriophages was performed basing on an alignment-free distance that estimates the similarity of two sequences (in our case entire viral proteomes) according to the average length of subsequences that are repeated in both of them (the ARS). The tree was built using the neighbor joining algorithm as implemented in [58].

See more details in Additional file 1: Section 3.12.

\section{Additional files}

Additional file 1: Supplementary results and material. (PDF $2568 \mathrm{~kb}$ )

Additional file 2: Full list of the analyzed viruses including their accession numbers and temporal labels of genes. (XLSX $111 \mathrm{~kb}$ )

\section{Abbreviations \\ AA: Amino acid; AAB: Amino acid bias; ARS: Average repetitive subsequences; CPB: Codon pair bias; CPS: Codon pair score; CUB: Codon usage bias; DBS: Davies-bouldin score; DNA: Deoxy ribonucleic acid; \\ DNTB: Dinucleotide bias; DNTS: Dinucleotide score; E.Coli: Escherichia coli; EMG: Exponentially modified gaussian; ENC: Effective number of codons; HIV: Human immunodeficiency virus; mRNA: Messenger ribonucleic acid; MTDR: Mean typical decoding rate; NFC: Normalized footprint count; NTB: Nucleotide bias; PCA: Principal component analysis; RNA: Ribonucleic acid; RSCF: Relative synonymous codons frequencies; RSCU: Relative synonymous codons usage; tAl: tRNA adaptation index; TDR: Typical decoding rate; tRNA: Transfer ribonucleic acid}

\section{Acknowledgements}

None.

\section{Funding}

E.G. is supported, in part, by a fellowship from the Edmond J. Safra Center for Bioinformatics at Tel-Aviv University. T.T. is grateful to the Minerva ARCHES award.

\section{Availability of data and materials}

The datasets supporting the conclusions of this article are included within the supplementary information in Additional file 1: Section 3.1 and Additional file 2.

Authors' contributions

$\mathrm{OM}, \mathrm{EG}, \mathrm{TT}$ analyzed the data and wrote the paper. All authors read and approved the final manuscript.

Ethics approval and consent to participate

Not applicable.

Consent for publication

Not applicable.

Competing interests

The authors declare that they have no competing interests.

\section{Publisher's Note}

Springer Nature remains neutral with regard to jurisdictional claims in published maps and institutional affiliations.

\section{Author details}

${ }^{1}$ Department of Biomedical Engineering, Tel-Aviv University, Ramat Aviv, Israel. ${ }^{2}$ SynVaccineLtd. Ramat Hachayal, Tel Aviv, Israel. ${ }^{3}$ Sagol School of Neuroscience, Tel-Aviv University, Ramat Aviv, Israel. 
Received: 5 July 2017 Accepted: 31 October 2017

Published online: 13 November 2017

\section{References}

1. Bonvicini F, Filippone C, Delbarba S, Manaresi E, Zerbini M, Musiani M, Gallinella G. Parvovirus B19 genome as a single, two-state replicative and transcriptional unit. Virology. 2006;347(2):447-54.

2. Fessler SP, Young CSH. Control of adenovirus early gene expression during the late phase of infection. J Virol. 1998;72(5):4049-56.

3. Gruffat $H$, Marchione R, Manet E. Herpesvirus late gene expression: a viralspecific pre-initiation complex is key. Front Microbiol. 2016;7:869.

4. Jia R, Liu XF, Tao MF, Kruhlak M, Guo M, Meyers C, Baker CC, Zheng ZM. Control of the Papillomavirus early-to-late Switch by differentially expressed SRp20. J Virol. 2009;83(1):167-80.

5. Liu Z, Carmichael GG. Polyoma-virus early-late switch - regulation of late rna accumulation by dna-replication. Proc Natl Acad Sci U S A. 1993;90(18):8494-8.

6. Nisole S, Saïb A. Early steps of retrovirus replicative cycle. Retrovirology. 2004;1(1):9.

7. Schiralli Lester GM, Henderson AJ. Mechanisms of HIV transcriptional regulation and their contribution to latency. Mol Biol Int. 2012;2012:11.

8. Yang $H, M a ~ Y$, Wang $Y$, Yang $H$, Shen $W$, Chen $X$. Transcription regulation mechanisms of bacteriophages. Bioengineered. 2014;5:300-4.

9. Levy JA, Fraenkel-Conrat H, Owens RA: Virology: prentice hall; 1994.

10. Saunders JBCaVA. Virology principles and applications. West Sussex: John Wiley \& Sons Ltd; 2007

11. Aragones L, Guix S, Ribes E, Bosch A, Pinto RM. Fine-tuning translation kinetics selection as the driving force of Codon usage bias in the hepatitis a virus Capsid. PLoS Pathog. 2010;6(3):e1000797.

12. Bahir I, Fromer M, Prat Y, Linial M. Viral adaptation to host: a proteomebased analysis of codon usage and amino acid preferences. Mol Syst Biol. 2009:5(1):311.

13. Bull JJ, Molineux IJ, Wilke CO. Slow fitness recovery in a Codon-modified viral genome. Mol Biol Evol. 2012;29(10):2997-3004.

14. Burns CC, Shaw J, Campagnoli R, Jorba J, Vincent A, Quay J, Kew O. Modulation of poliovirus replicative fitness in HeLa cells by deoptimization of synonymous codon usage in the capsid region. J Virol. 2006;80(7):3259-72.

15. Cai MS, Cheng AC, Wang MS, Zhao LC, Zhu DK, Luo QH, Liu F, Chen XY Characterization of synonymous Codon usage bias in the duck plague virus UL35 gene. Intervirology. 2009;52(5):266-78.

16. Das S, Paul S, Dutta C. Synonymous codon usage in adenoviruses: influence of mutation, selection and protein hydropathy. Virus Res. 2006;117(2):227-36

17. Goz E, Mioduser O, Diament A, Tuller T. Evidence of translation efficiency adaptation of the coding regions of the bacteriophage lambda. DNA Res. 2017;24(4):333-42.

18. Jia RY, Cheng AC, Wang MS, Xin HY, Guo YF, Zhu DK, Qi XF, Zhao LC, Ge H, Chen XY. Analysis of synonymous codon usage in the UL24 gene of duck enteritis virus. Virus Genes. 2009;38(1):96-103.

19. Liu YS, Zhou JH, Chen HT, Ma LN, Ding YZ, Wang M, Zhang J. Analysis of synonymous codon usage in porcine reproductive and respiratory syndrome virus. Infect Genet Evol. 2010;10(6):797-803.

20. Liu YS, Zhou JH, Chen HT, Ma LN, Pejsak Z, Ding YZ, Zhang J. The characteristics of the synonymous codon usage in enterovirus 71 virus and the effects of host on the virus in codon usage pattern. Infect Genet Evol. 2011;11(5):1168-73.

21. Ma MR, Ha XQ, Ling H, Wang ML, Zhang FX, Zhang SD, Li G, Yan W. The characteristics of the synonymous codon usage in hepatitis $B$ virus and the effects of host on the virus in codon usage pattern. Virol J. 2011;8(1):544.

22. Michely S, Toulza E, Subirana L, John U, Cognat V, Marechal-Drouard L, Grimsley N, Moreau H, Piganeau G. Evolution of Codon usage in the smallest photosynthetic eukaryotes and their Giant viruses. Genome Biol Evol. 2013;5(5):848-59.

23. RoyChoudhury S, Mukherjee D. A detailed comparative analysis on the overall codon usage pattern in herpesviruses. Virus Res. 2010;148(1-2): $31-43$.

24. Sau K, Gupta SK, Sau S, Ghosh TC. Synonymous codon usage bias in 16 Staphylococcus Aureus phages: implication in phage therapy. Virus Res. 2005;113(2):123-31.
25. Sharp PM, Bailes E, Grocock RJ, Peden JF, Sockett RE. Variation in the strength of selected codon usage bias among bacteria. Nucleic Acids Res. 2005;33(4):1141-53.

26. Su MW, Lin HM, Yuan HS, Chu WC. Categorizing host-dependent RNA viruses by principal component analysis of their Codon usage preferences. J Comput Biol. 2009;16(11):1539-47.

27. Tao P, Dai L, Luo MC, Tang FQ, Tien P, Pan ZS. Analysis of synonymous codon usage in classical swine fever virus. Virus Genes. 2009;38(1):104-12.

28. Tsai CT, Lin CH, Chang CY. Analysis of codon usage bias and base compositional constraints in iridovirus genomes. Virus Res. 2007;126(1-2): 196-206.

29. Wong EHM, Smith DK, Rabadan R, Peiris M, LLM P. Codon usage bias and the evolution of influenza a viruses. Codon usage biases of influenza virus. BMC Evol Biol. 2010;10(1):253.

30. Zhang ZC, Dai W, Wang Y, Lu CP, Fan HJ. Analysis of synonymous codon usage patterns in torque teno sus virus 1 (TTSuV1). Arch Virol. 2013;158(1): $145-54$

31. Zhao KN, Gru WY, Fang NX, Saunders NA, Frazer $\mathbb{H}$. Gene codon composition determines differentiation-dependent expression of a viral capsid gene in keratinocytes in vitro and in vivo. Mol Cell Biol. 2005;25(19): 8643-55.

32. Zhong J, Li Y, Zhao S, Liu S, Zhang Z. Mutation pressure shapes codon usage in the GC-rich genome of foot-and-mouth disease virus. Virus Genes. 2007;35(3):767-76

33. Zhou JH, Zhang J, Chen HT, Ma LN, Liu YS. Analysis of synonymous codon usage in foot-and-mouth disease virus. Vet Res Commun. 2010; 34(4):393-404

34. Novella IS, Zarate S, Metzgar D, Ebendick-Corpus BE. Positive selection of synonymous mutations in vesicular stomatitis virus. J Mol Biol. 2004;342(5): 1415-21.

35. Spencer PS, Barral JM. Genetic code redundancy and its influence on the encoded polypeptides. Comput Struct Biotechnol J. 2012;1(1):1-8.

36. Coleman JR, Papamichail D, Skiena S, Futcher B, Wimmer E, Mueller S. Virus attenuation by genome-scale changes in codon pair bias. Science. 2008; 320(5884):1784-7

37. Greenbaum BD, Levine AJ, Bhanot G, Rabadan R. Patterns of evolution and host gene mimicry in influenza and other RNA viruses. PLoS Pathog. 2008; 4(6):e1000079.

38. Karlin S, Doerfler W, Cardon LR. Why is CpG suppressed in the genomes of virtually all small eukaryotic viruses but not in those of large eukaryotic viruses? J Virol. 1994;68(5):2889-97.

39. Rima BK, McFerran NV. Dinucleotide and stop codon frequencies in singlestranded RNA viruses. J Gen Virol. 1997:78(11):2859-70.

40. Yakovchuk P, Protozanova E, Frank-Kamenetskii MD. Base-stacking and basepairing contributions into thermal stability of the DNA double helix. Nucleic Acids Res. 2006;34(2):564-74.

41. Cheng XF, Virk N, Chen W, Ji SQ, Ji SX, Sun YQ, Wu XY. CpG usage in RNA viruses: data and hypotheses. PLoS One. 2013;8(9):e74109.

42. Wang AHJ, Hakoshima T, Vandermarel G, Vanboom JH, Rich A. At base-pairs are less stable than Gc Base-pairs in Z-Dna - the crystal-structure of D(M5cgtam5cg). Cell. 1984;37(1):321-31.

43. Zur $\mathrm{H}$, Tuller $\mathrm{T}$. Strong association between mRNA folding strength and protein abundance in S. Cerevisiae. EMBO Rep. 2012:13(3):272-7.

44. Mortimer SA, Kidwell MA, Doudna JA. Insights into RNA structure and function from genome-wide studies. Nat Rev Genet. 2014;15(7):469.

45. Xia XH. Maximizing transcription efficiency causes codon usage bias. Genetics. 1996;144(3):1309-20.

46. Cohen E, Zafrir Z, and Tuller T. A Code for Transcription Elongation Speed. To appear in RNA Biology. 2017.

47. Zhang $G$, Ignatova $Z$. Folding at the birth of the nascent chain: coordinating translation with co-translational folding. Curr Opin Struct Biol. 2011:21(1):25-31.

48. Akashi H, Gojobori T. Metabolic efficiency and amino acid composition in the proteomes of Escherichia Coli and Bacillus Subtilis. Proc Natl Acad Sci U S A. 2002;99(6):3695-700

49. Davies DL, Bouldin DW. A cluster separation measure. IEEE Trans Pattern Anal Mach Intell. 1979;1(2):224-7.

50. Sharp PM, Li WH. An evolutionary perspective on synonymous Codon usage in unicellular organisms. J Mol Evol. 1986;24(1-2):28-38.

51. Sabi R, Tuller T. Modelling the efficiency of Codon-tRNA interactions based on Codon usage bias. DNA Res. 2014;21(5):511-25. 
52. Sabi R, Daniel RV, Tuller T. stAl(calc): tRNA adaptation index calculator based on species-specific weights. Bioinformatics. 2017;33(4):589-91.

53. Wright F. The effective number of Codons used in a gene. Gene. 1990; 87(1):23-9.

54. Karlin S. Global dinucleotide signatures and analysis of genomic heterogeneity. Curr Opin Microbiol. 1998;1(5):598-610.

55. Liu XQ, Jiang HF, Gu ZL, Roberts JW. High-resolution view of bacteriophage lambda gene expression by ribosome profiling. Proc Natl Acad Sci U S A. 2013;110(29):11928-33.

56. Dana A, Tuller T. The effect of tRNA levels on decoding times of mRNA codons. Nucleic Acids Res. 2014;42(14):9171-81.

57. Ulitsky I, Burstein D, Tuller T, Chor B. The average common substring approach to phylogenomic reconstruction. J Comput Biol. 2006;13(2):336-50.

58. Felsenstein J. PHYLIP - phylogeny inference package (version 3.2). Cladistics. 989:5(2):163-6.

Submit your next manuscript to BioMed Central and we will help you at every step:

- We accept pre-submission inquiries

- Our selector tool helps you to find the most relevant journal

- We provide round the clock customer support

- Convenient online submission

- Thorough peer review

- Inclusion in PubMed and all major indexing services

- Maximum visibility for your research

Submit your manuscript at www.biomedcentral.com/submit 